\title{
Nuclear distribution of eIF3g and its interacting nuclear proteins in breast cancer cells
}

\author{
QIAOLI ZHENG ${ }^{1,2}$, HAO LIU $^{3}$, JINGJIA YE ${ }^{1}$, HUI ZHANG ${ }^{4}$, ZHENYU JIA $^{3}$ and JIANG CAO ${ }^{1}$ \\ ${ }^{1}$ Clinical Research Center, The Second Affiliated Hospital, School of Medicine, Zhejiang University, Hangzhou, \\ Zhejiang 310009; ${ }^{2}$ Department of Dermatology, Sir Run Run Shaw Hospital, School of Medicine, Zhejiang University, \\ Hangzhou, Zhejiang 310016; ${ }^{3}$ Institute of Occupational Diseases, Zhejiang Academy of Medical Sciences, Hangzhou, \\ Zhejiang 310007; ${ }^{4}$ Department of Clinical Laboratory, Hangzhou First People's Hospital, \\ Hangzhou, Zhejiang 310006, P.R. China
}

Received March 11, 2015; Accepted January 11, 2016

DOI: $10.3892 / \mathrm{mmr} .2016 .4935$

\begin{abstract}
Eukaryotic translation initiation factor 3 subunit $\mathrm{g}$ (eIF3g) is a core subunit of the eukaryotic translation initiation factor 3 complex, and is important in the initiation of translation. It is also involved in caspase-mediated apoptosis, and is upregulated in multidrug-resistant cancer cells. In the present study, the nuclear distribution of eIF3g was determined by performing co-immunoprecipitation of proteins that potentially interact with eIF3g in the nucleus. Mass spectrometry characterization showed that three proteins, heterogeneous nuclear ribonucleoprotein U/scaffold attachment factor A, HSZFP36/zinc finger protein 823 and $\beta$-actin, were among the candidate eIF3g-interacting proteins in the nucleus. The protein-protein interaction was further confirmed by cross-linking and a glutathione S-transferase pull-down assay, followed by western blotting. The co-localization of these proteins was determined by confocal microscopy. These
\end{abstract}

Correspondence to: Dr Jiang Cao, Clinical Research Center, The Second Affiliated Hospital, School of Medicine, Zhejiang University, 88 Jiefang Road, Hangzhou, Zhejiang 310009, P.R. China E-mail: caoj@zju.edu.cn

Dr Zhenyu Jia, Institute of Occupational Diseases, Zhejiang Academy of Medical Sciences, 182 Tianmushan Road, Hangzhou, Zhejiang 310007, P.R. China

E-mail: zhengyujia@yahoo.com

Abbreviations: CaMV, cauliflower mosaic virus; co-IP, co-immunoprecipitation; eIF, eukaryotic translation initiation factor; FBS, fetal bovine serum; GAPDH, glyceraldehyde-3-phosphate dehydrogenase; hnRNP U/SAF-A, heterogeneous nuclear ribonucleoprotein U/scaffold attachment factor A; PBS, phosphate buffer saline; PVDF, polyvinylidene fluoride; RRM, RNA recognition motif; TAV, transactivator/viroplasmin; ZFP823, zinc finger protein $823 ; 4.1 \mathrm{R}$, erythroid protein 4.1

Key words: eukaryotic translation initiation factor 3 subunit g, cancer, heterogeneous nuclear ribonucleoprotein U, HSZFP36, $\beta$-actin, nucleus findings provide novel insight into the possible functions of eIF3g in the nucleus and serves as an important first step for further investigation of the roles of eIF3g in cancer development.

\section{Introduction}

Translation initiation is one of the key regulatory steps for protein synthesis in mammalian cells, and is performed by eukaryotic translation initiation factors (eIFs). Eukaryotic translation initiation factor 3 (eIF3) mediates the interaction between the $43 \mathrm{~S}$ pre-initiation complex and the eIF4F-bound mRNA clients (1). eIF3 is comprised of 13 subunits (eIF3a-m; $\sim 700-800 \mathrm{kDa}$ total mass), and each of these contributes to the function (2). Similarly, each subunit of eIF3 may exert functions in biological processes other than translation initiation. For example, the eIF3a subunit is associated with cell cycle regulation, and the eIF3e subunit may be involved in mitosis and segregation (2). The key position of eIF3 in the mechanism of translation initiation and other functions of its subunits contributes to the important roles of eIF3 and its subunits in various aspects of cellular activities under physiological and pathological conditions, including cancer $(2,3)$. The aberrant expression of certain subunits of eIF3 in several types of cancer has been associated with the phenotypes of cancer cells, including malignant growth, proliferation, invasion, metastasis and clinical parameters, including disease staging and prognosis (4-9).

eIF3g is a core eIF3 subunit, which is known to be involved in the process of translation reinitiation (2). Previous studies have reported that eIF3g is also involved in the cytoskeletal network and caspase-mediated apoptosis $(10,11)$. Our previous study demonstrated that eIF3g was overexpressed in an adriamycin-resistant human erythroleukemia cell line (12). In addition, a positive correlation was observed between the overexpression of eIF3g and lymph node metastasis in a pilot study performed to evaluate the clinical significance of eIF3g overexpression in breast cancer (data not shown). These results led to the hypotheses that the aberrant expression of eIF3g may be involved in the development and/or the progression of cancer by mediating aberrant apoptosis and multidrug resistance. It is 
important to test these hypotheses and to examine the possible mechanisms.

Our previous bioinformatics investigation to identify functional domains of eIF3g other than those for translation initiation revealed that eIF3g may have a nuclear localization sequence. Therefore, the present study aimed to examine the nuclear localization of eIF3g and to investigate the possible functions of nuclear eIF3g, as well as its interacting nuclear proteins, in order elucidate the mechanisms underlying the effect of eIF3g on the development/progression of breast cancer.

\section{Materials and methods}

Bioinformatics analysis. Several online bioinformatics tools were used to predict the subcellular distribution of eIF3g, including WoLF PSORT (http://wolfpsort.org), PROST II Prediction (http://psort.hgc.jp/form2.html), Subnuclear Compartments Prediction System (http://array.bioengr. uic.edu/subnuclear.htm) and PredictProtein (https://www. predictprotein.org). The sequence-based protein function was predicted using the online tool, PredictProtein (https://www. predictprotein.org). Protein sequence data were obtained from the National Center for Biotechnology Information Protein database (http://www.ncbi.nlm.nih.gov/protein). Gene Ontology terms are organized in two different ontologies, Molecular Function Ontology and Biological Process Ontology (http://geneontology.org/).

Overexpression of eIF3g in Bcap37 human breast cancer cells. The human breast cancer cell line, Bcap37 (The Cell Bank of Type Culture Collection of the Chinese Academy of Sciences, Shanghai, China), with doxycycline-inducible overexpression of eIF3g (Bcap37/Tet-on-eIF3g) was used as previously described (13). Briefly, the human breast cancer Bcap37 cells were cultured in RPMI 1640 medium (Boster Biological Technology) supplemented with $10 \%$ fetal bovine serum (FBS; Gbico; Thermo Fisher Scientific, Inc., Waltham, MA, USA) or 10\% Tet-approved FBS (Clontech Laboratories, Mountain View, CA, USA) in a humidified $37^{\circ} \mathrm{C}$ incubator. The full length eIF3g cDNA in the pLVX-Tight-Puro vector (Clontech Laboratories) was used to produce the lentivirus, following packaging with a Lenti-X ${ }^{\mathrm{TM}}$ Tet-On Avanced Inducible Expression System (Clontech Laboratories), according to the manufacturer's protocol. HEK293T cells $\left(8 \times 10^{6}\right.$ per dish; American Type Culture Collection, Manassas, VA, USA) were cultured in Dulbecco's modified Eagle's medium (DMEM) with high glucose (Boster Biological Technology), supplemented with $10 \%$ FBS, and used for transfection. The lentivirus-containing supernatant was collected. The Bcap37 cells $\left(6 \times 10^{5}\right.$ per dish) were infected with the lentivirus in a 6-well plate. Geneticin $(250 \mu \mathrm{g} / \mathrm{ml}$; Amresco LLC, Solon, $\mathrm{OH}, \mathrm{USA})$ and puromycin $(0.25 \mu \mathrm{g} / \mathrm{ml}$; Sigma-Aldrich, St. Louis, MO, USA) were used to select and maintain stable transfectants. Doxycycline ( $1 \mu \mathrm{g} / \mathrm{ml}$; Sigma-Aldrich) was used to induce the overexpression of eIF3g.

Rapid, efficient and practical method for cell fractionation. Fractionation was performed to separate the cytosolic fraction and nuclear fraction of the cells (14). Briefly, the trypsinized cells were washed once for $1 \mathrm{~min}$ with ice-cold phosphate-buffered saline (PBS) and lysed with $1 \mu 1$ ice-cold $0.1 \%$ NP-40 in PBS. A small volume $(300 \mu 1)$ of the lysate was saved as 'whole cell lysate'. The remaining lysate was centrifuged at $15,000 \times \mathrm{g}\left(4^{\circ} \mathrm{C}\right)$ for $5 \mathrm{~min}$, and the supernatant was collected as the 'cytosolic fraction'. The pelleted 'nuclear fraction' was further lysed in radioimmunoprecipitation assay (RIPA) buffer [50 mM Tris-HCl (pH 7.4), 1\% NP-40, 0.5\% sodium deoxycholate, $150 \mathrm{mM} \mathrm{NaCl}$ and $0.1 \%$ SDS], containing protease inhibitor cocktail tablets (Roche Diagnostics, Basel, Switzerland). For crosslinking experiments, the modified RIPA buffer (1X PBS, 1\% NP-40, 0.5\% sodium deoxycholate, $150 \mathrm{mM} \mathrm{NaCl}$ and $0.1 \%$ SDS) was used. The lysate was centrifuged at $15,000 \times \mathrm{g}\left(4^{\circ} \mathrm{C}\right)$ for $15 \mathrm{~min}$ and the supernatant was collected as the 'nuclear fraction'.

Western blot analysis. The cells were lysed in RIPA buffer and the lysate was centrifuged at $15,000 \times \mathrm{g}$ for $15 \mathrm{~min}$ at $4^{\circ} \mathrm{C}$. The protein supernatant was quantified using a DC Protein Assay kit (Bio-Rad Laboratories, Inc., Hercules, CA, USA). The cell lysates and protein samples, prepared in reducing sample buffer [50 mM Tris- $\mathrm{HCl}$ ( $\mathrm{pH}$ 6.8), $2 \%$ SDS, $0.01 \%$ Bromophenol Blue, $10 \%$ glycerol and $50 \mathrm{mM}$ DTT] were subjected to SDS-PAGE and transferred onto polyvinylidene fluoride $(0.2 \mu \mathrm{m}$ PVDF) membranes. Following blocking with $10 \%$ nonfat milk for $1 \mathrm{~h}$, the membranes were incubated with the following antibodies: Polyclonal rabbit anti-human eIF3g (1:10,000; Bethyl Laboratories, Inc., Montgomery, TX, USA; cat. no. A301-757A), polyclonal rabbit anti-human hnRNP U (1:1,000; Abcam Cambridge, UK; cat. no. ab20666), polyclonal goat anti-human HSZFP36 (1:1,000; Santa Cruz Biotechnology, Inc., Santa Cruz, CA, USA; cat. no. sc-247189), polyclonal goat anti-human $\beta$-actin (1:1,000; Santa Cruz Biotechnology, Inc.; cat. no. sc-1615), polyclonal rabbit anti-human Histone H4 (cat. no. 07-108; 1:1,000; EMD Millipore, Billerica, MA, USA) overnight at $4^{\circ} \mathrm{C}$. This was followed by incubation with secondary peroxidase-conjugated goat anti-rabbit $\operatorname{IgG}(1: 5,000 ; \mathrm{ZB}-2301)$ and rabbit anti-goat IgG (1:5,000; ZB-2306) (Zhongshan Golden Bridge Biotechnology, Co, Ltd, Beijing, China) or horseradish peroxidase-conjugated polyclonal rabbit anti-human GAPDH (cat. no. sc-25778; 1:1,000; Santa Cruz Biotechnology, Inc.) for $2 \mathrm{~h}$ at room temperature. Enhanced chemiluminescence substrates (EMD Millipore) were then applied and signals were detected using a chemiluminescence imaging system (ChemiDoc $^{\text {TM }}$ MP Imaging System; Bio-Rad Laboratories, Inc.).

Co-immunoprecipitation (co-IP). The nuclear protein $(200 \mu \mathrm{g})$ was incubated with the primary antibodies mentioned above (5 $\mu \mathrm{g}$ anti-eIF3g, $5 \mu \mathrm{g}$ anti-hnRNPU, $5 \mu \mathrm{g}$ anti-HSZFP36 or $5 \mu \mathrm{g}$ anti- $\beta$-actin) and $50 \mu \mathrm{l}$ protein $\mathrm{A} / \mathrm{G}$ PLUS-Agarose (Santa Cruz Biotechnology, Inc.) on a rotating device (Tube Tumbler Rotating Mixer SBS550-2; Select BioProducts, Edison, NJ, USA) at $4^{\circ} \mathrm{C}$ overnight. Normal rabbit serum ( $2 \mu \mathrm{l}$ ) was used as a control group. Following centrifugation at $5,000 \mathrm{x} \mathrm{g}$ for $10 \mathrm{~min}$ at $4^{\circ} \mathrm{C}$, the co-immunoprecipitated proteins were washed with PBS four times (5 min each time), treated with reducing sample buffer and subjected to $10 \%$ SDS-PAGE. Following electrophoresis of the proteins, the 
gel was stained using $50 \mathrm{ml}$ Bio-safe Coomassie blue stain (Bio-Rad Laboratories, Inc.) for $1 \mathrm{~h}$ at room temperature with gentle agitation, followed by destaining in distilled water for $>2 \mathrm{~h}$ until the protein bands were clearly visible. The protein bands were excised and placed into microtubes, and were sent for mass spectrometry characterization of proteins by service provider (Institute of Biomedical Sciences, Fudan University, Shanghai, China).

In vitro crosslinking. The separated nuclear fraction was lysed with modified RIPA buffer. DSS (Thermo Fisher Scientific, Inc.; spacer arm, $11.4 \AA$ ) was added to the nuclear fraction to a final concentration of $5 \mathrm{mM}$ and incubated at room temperature for $30 \mathrm{~min}$. To quench the reaction, quenching buffer (1 M Tris- $\mathrm{Cl}$; $\mathrm{pH} 7.5)$ was added to a final concentration of 20-50 mM Tris (Bio-Rad Laboratories, Inc.) at room temperature for $15 \mathrm{~min}$. The mixtures were then prepared in SDS-PAGE loading buffer for western blot analysis.

Glutathione S-transferase (GST) pull-down assay. The full length coding sequence of eIF3g was inserted into the pGEX-5X-2 vector (Pharmacia Biotech, Tokyo, Japan), in-frame with the GST open reading frame, which led to the production of soluble GST-eIF3g fusion protein when transformed into Escherichia coli DH5a (Takara Bio Inc., Otsu, Shiga, Japan) and induced by $0.1 \mathrm{mM}$ isopropyl- $\beta-\mathrm{D}-1$ -thiogalactopyranoside (Amresco LLC) for $4 \mathrm{~h}$ at $37^{\circ} \mathrm{C}$. For the GST pull-down assays, the GST or GST-eIF3g proteins expressed in $E$. coli were purified using magnetic glutathione-beads (Promega Corp., Madison, WI, USA), according to the manufacturer's protocol. Briefly, bacteria were collected following culture by centrifugation at 5,000 $\mathrm{x}$ g for $15 \mathrm{~min}$ at $4^{\circ} \mathrm{C}$, resuspension in $3 \mathrm{ml}$ ice-cold PBS and lysis by sonication on ice. The lysate was centrifuged at $5,000 \mathrm{x} \mathrm{g}$, for $10 \mathrm{~min}$ at $4^{\circ} \mathrm{C}$ to remove the cell debris, and $100 \mu \mathrm{l}$ magnetic glutathione beads were added to the supernatant for binding of the GST/GST-eIF3g protein. The beads were washed three times (5 min each time) with PBS and then incubated with the nuclear lysates from the Bcap37 cells with rotation for $30 \mathrm{~min}$ at room temperature. The beads were then washed at least three times with PBS (5 min each time); bound proteins were resolved by $8 \%$ SDS-PAGE and detected by western blotting.

Immunofluorescent staining. The Bcap37/Tet-on-eIF3g and Bcap37 cells were seeded separately into a 6 -well plate with a glass-bottomed dish at a density of $2 \times 10^{4}$ cells/dish and treated with doxycycline for 3 days at $37^{\circ} \mathrm{C}$. The cells were then washed with PBS three times (10 min each time), fixed with $4 \%$ paraformaldehyde (Shanghai Ling Feng Chemicals Co., Ltd., Shanghai, China) for $10 \mathrm{~min}$, and permeabilized with $0.1 \%$ Triton X-100 (Bio Basic Inc., Markham, ON, Canada) for $10 \mathrm{~min}$. Following washing with PBS three times, the cells were blocked with 5\% goat serum (Zhongshan Golden Bridge Biotechnology Co., Ltd.) and incubated with anti-eIF3g (1:400), anti-hnRNP U (1:200), anti-HSZFP36 (1:200) or anti- $\beta$-actin (1:200) primary antibodies overnight at $4^{\circ} \mathrm{C}$. The cells were then washed with PBS three times $(10 \mathrm{~min}$ each time) and further incubated with following secondary antibodies: Fluorescein isothiocyanate-conjugated donkey
Table I. Predicted subcellular distribution of eIF3g using online bioinformatics tools.

\begin{tabular}{ll} 
Bioinformatics tool & Distribution \\
\hline WoLF PSORT & $\begin{array}{l}\text { Cytoplasm } \\
\text { and nucleus }\end{array}$ \\
PROST II Prediction & Nucleus \\
Subnuclear Compartments Prediction System & Nucleus \\
PredictProtein & Nucleus \\
\hline
\end{tabular}

Websites: WoLF PSORT, http://wolfpsort.org; PROST II Prediction, http://psort.hgc.jp/form2.html; Subnuclear Compartments Prediction System, http://array.bioengr.uic.edu/subnuclear.htm; PredictProtein, https://www.predictprotein.org.

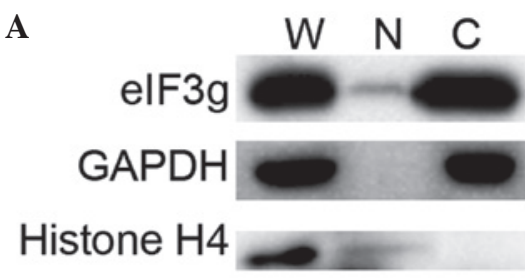

B $\quad$ elF3g

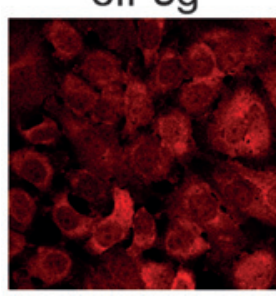

DAPI

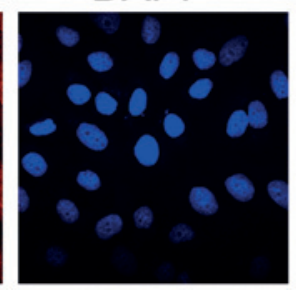

Merged

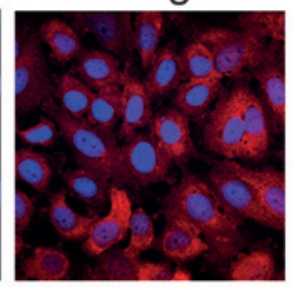

Figure 1. Nuclear localization of eIF3g. (A) Results of fractionation of theBcap37/Tet-on-eIF3g cells. GAPDH was used as the cytoplasmic marker and Histone $\mathrm{H} 4$ was used as the nuclear marker. The proteins samples were subjected to $12 \%$ SDS-PAGE, transferred onto a polyvinylidene fluoride membrane and probed by specific antibodies. (B) Immunofluorescent staining was performed. Confocal microscope images for eIF3g are shown (magnification, x100). eIF3g, eukaryotic translation initiation factor 3 subunit $\mathrm{g}$; W, whole cell lysate; $\mathrm{C}$, cytoplasmic fraction; $\mathrm{N}$, nuclear fraction.

anti-rabbit IgG (1:500; 711-095-152) and/or tetramethylrhodamine-conjugated donkey anti-goat IgG (1:500; 705-025-003) (Jackson ImmunoResearch Laboratories, Inc., West Grove, PA, USA). The nuclei were stained with $200 \mu \mathrm{g} / \mathrm{ml}$ DAPI (Sigma-Aldrich). The stained cells were observed under a confocal microscope (LSM710; Carl Zeiss, Jena, Germany).

\section{Results}

Nuclear localization of eIF3g. The present study hypothesized that eIF3g may link other biological processes to the initiation of translation. To confirm this, in silico analysis and subcellular fractionation was performed to examine the functional domains and subcellular distribution of eIF3g. As shown in Tables I and II, bioinformatics analyses using state-of-art online tools predicted that eIF3g has a nuclear distribution for potential functions. 
Table II. Predicted function of eukaryotic translation initiation factor 3 subunit $\mathrm{g}$ in the nucleus.

\begin{tabular}{llr}
\hline GO ID & \multicolumn{1}{c}{ Molecular function/biological process } & Reliability (\%) \\
\hline GO:0003677 & DNA binding & 2 \\
GO:0003697 & Single-stranded DNA binding & 2 \\
GO:0003690 & Double-stranded DNA binding & 1 \\
GO:0006396 & RNA processing & 14 \\
GO:0051028 & mRNA transport & 9 \\
GO:0045449 & Regulation of transcription & 8 \\
GO:0060211 & Regulation of nuclear-transcribed mRNA poly (A) tail shortening & 8 \\
GO:0048255 & mRNA stabilization & 8 \\
GO:0031047 & Gene silencing by RNA & 8 \\
GO:0000184 & Nuclear-transcribed mRNA catabolic process, nonsense-mediated decay & 8 \\
GO:0060212 & Negative regulation of nuclear-transcribed mRNA poly (A) tail shortening & 8 \\
GO:0000398 & Nuclear mRNA splicing, via spliceosome & 8 \\
GO:0006350 & Transcription & 8
\end{tabular}

GO, Gene Ontology.

To verify this prediction, the present study examined the expression of eIF3g in Bcap37 breast cancer cells by western blotting in a preliminary experiment, and the results showed a weak, but distinct band of eIF3g in the nuclear protein fraction (data not shown). The Bcap37/Tet-on-eIF3g cells with inducible overexpression of eIF3g were then used in subsequent investigations. The cells were harvested through subcellular fractionation of the cytosolic and the nuclear proteins of breast cancer cells expressing eIF3g (Bcap37/Tet-on-eIF3g). As shown in Fig. 1A, the use of this method successfully separated the cytosolic and the nuclear fractions, confirmed by detection of nuclear protein, Histone H4, in the whole cell lysate and nuclear fraction, but not in the cytoplasmic fraction. By contrast, the cytoplasmic protein, GAPDH, was detected in the whole cell lysate and cytosolic fraction, but not in the nuclear fraction. Although eIF3g was abundant in the cytosolic fraction, it was also detected in the nuclear fraction. Immunofluorescent staining confirmed the nuclear distribution of eIF3g (Fig. 1B). This was a novel finding, considering eIF3g was originally identified as a cytoplasmic protein and as a component of the translation initiation machinery (2).

Identification of nuclear proteins potentially interacting with $e I F 3 g$. Functional nuclear proteins exert their functions via binding directly to DNA or RNA to regulate gene expression or RNA processing, or by forming a protein complex to cooperate with other nuclear proteins (15). The present study focused primarily on identifying the possible nuclear proteins, which may interact with eIF3g. The nuclear fraction of the eIF3g-expressing cells was isolated, and co-IP was formed with anti-eIF3g antibody to pull down proteins that physically interacted with eIF3g. The immunoprecipitated proteins were separated by SDS-PAGE and visualized by Coomassie blue staining (Fig. 2), and four bands were selected for subsequent mass spectrometry for protein characterization, excluding non-specific bands. As shown in Table III, the proteins were heterogeneous nuclear ribonucleoprotein U/scaffold

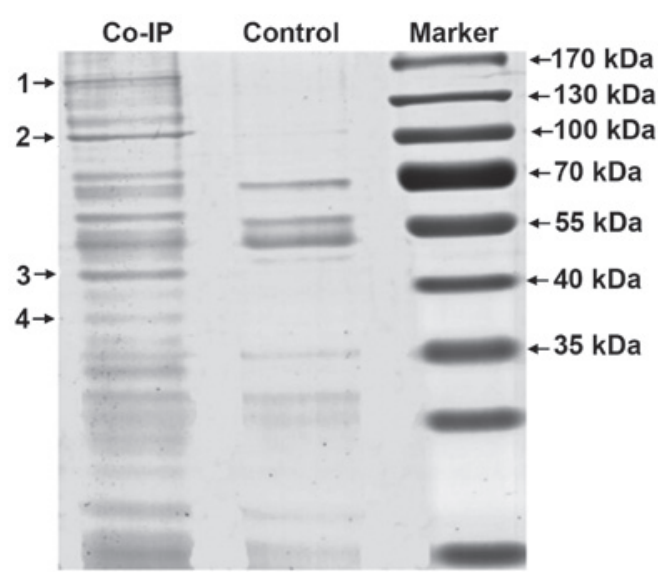

Figure 2. co-IP of proteins with anti-eIF3g antibody in the nuclear fraction of Bcap37/Tet-on-eIF3g cells. The nuclear protein $(200 \mu \mathrm{g})$ was incubated with the $5 \mu \mathrm{g}$ anti-eIF3g antibody and $50 \mu \mathrm{l}$ protein A/G PLUS-Agarose. Bands that were clearly present and with clear edges for excision in the co-IP sample but absent in the control sample (bands 1,2,3 and 4) were selected for protein characterization by mass spectrometry analysis. co-IP, co-immunoprecipitation; eIF3g, eukaryotic translation initiation factor 3 subunit $g$.

attachment factor A (hnRNP U/SAF-A), HSZFP36/zinc finger protein 823 (ZFP823), $\beta$-actin and eIF3a, respectively.

Confirmation of the interaction between eIF3g and hnRNP $U$, HSZFP36 and $\beta$-actin. The present study confirmed the physical interaction between eIF3g and its potential partners, hnRNP U, HSZFP36 and $\beta$-actin, by performing co-IP and in vitro crosslinking in combination with western blotting. As shown in Fig. 3A, eIF3g was co-immunoprecipitated from the nuclear fractions using antibodies against hnRNP U, HSZFP36 and $\beta$-actin, respectively. These proteins were also co-immunoprecipitated using antibody against eIF3g (Fig. 3B-D).

The results of the western blotting for the crosslinked proteins are shown in Fig. 3E, in which two specific bands were detected by the anti-eIF3g antibody; these bands were 
Table III. Mass spectrometry data of the proteins pulled down by eukaryotic translation initiation factor 3 subunit $\mathrm{g}$.

\begin{tabular}{|c|c|c|c|c|c|}
\hline Spot & $\begin{array}{l}\text { Accession } \\
\text { number }\end{array}$ & Protein & $\begin{array}{l}\text { Predicted } \\
\text { mass (Da) }\end{array}$ & Score & Function in nucleus \\
\hline 1 & gil6685537 & $\begin{array}{l}\text { Eukaryotic translation initiation } \\
\text { factor } 3 \text { subunit A (Homo sapiens) }\end{array}$ & 166,468 & 547 & Not reported \\
\hline 2 & gil3202000 & $\begin{array}{l}\text { Scaffold attachment factor } \mathrm{A} \\
\text { (Homo sapiens) }\end{array}$ & 90,423 & 320 & $\begin{array}{l}\text { DNA repair, telomere length } \\
\text { regulation, chromatin } \\
\text { remodeling, transcription } \\
\text { regulation }\end{array}$ \\
\hline 3 & gil148887464 & Zinc finger protein 823 (Homo sapiens) & 70,225 & 53 & Transcriptional regulation \\
\hline 4 & gil14250401 & $\beta$-actin (Homo sapiens) & 40,978 & 193 & $\begin{array}{l}\text { Chromatin remodeling, } \\
\text { transcription regulation } \\
\text { nucleocytoplasmic trafficking }\end{array}$ \\
\hline
\end{tabular}
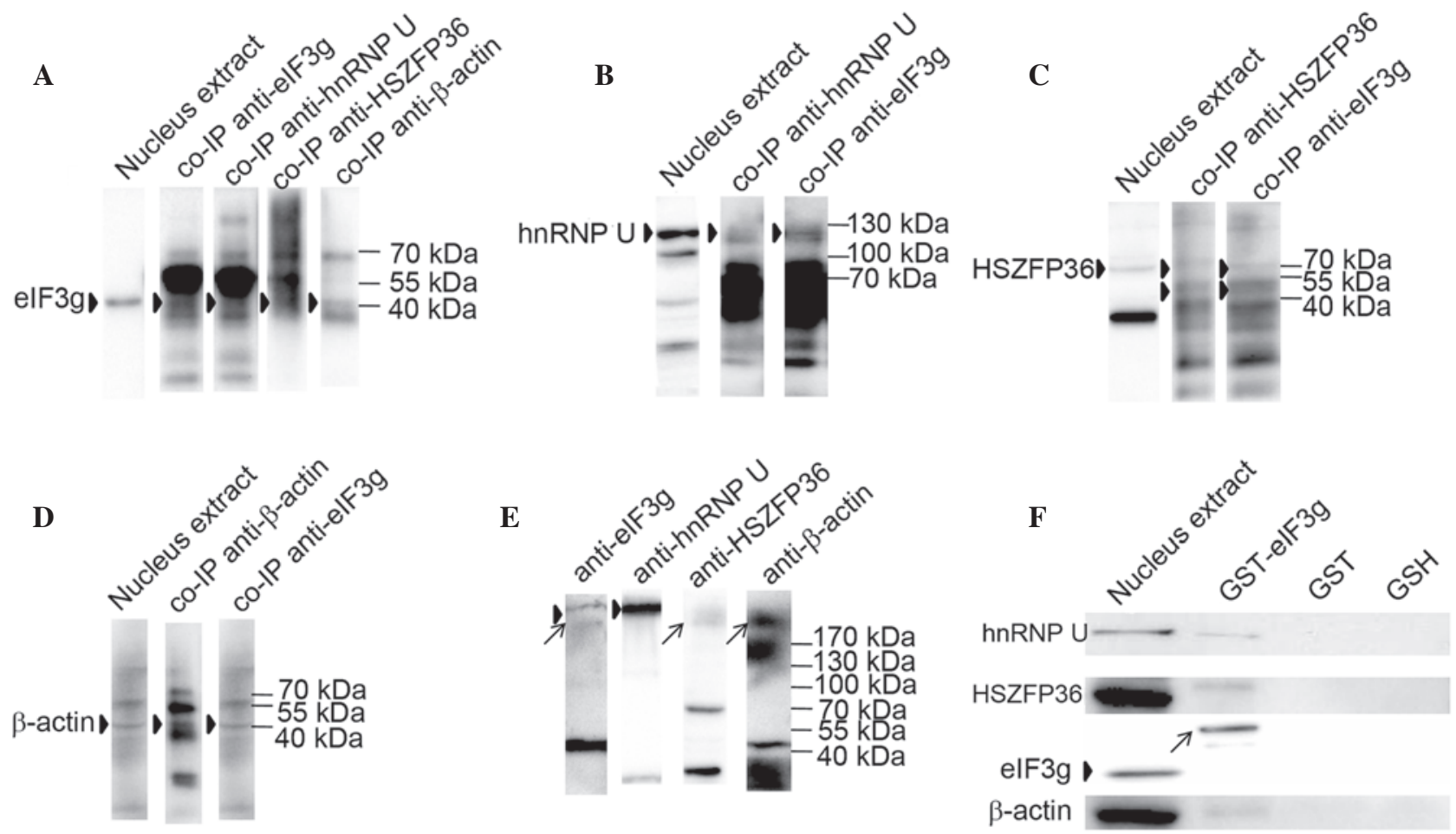

Figure 3. eIF3g interacts with hnRNP U, HSZFP36 and $\beta$-actin. (A) Nuclear extracts of Bcap37/Tet-on-eIF3g cells were subjected to immunoprecipitation with anti-eIF3g $(5 \mu \mathrm{g})$, anti-hnRNP U $(5 \mu \mathrm{g})$, anti-HSZFP36 $(5 \mu \mathrm{g})$ and anti- $\beta$-actin $(5 \mu \mathrm{g})$ antibodies, respectively. Precipitated proteins were resolved by $10 \%$ SDS-PAGE and analyzed by western blotting with anti-eIF3g antibody. Arrowheads indicate eIF3g. (B) Anti-eIF3g antibody-immunoprecipitated proteins were analyzed by western blotting with anti-hnRNP U antibody. Arrowheads indicate hnRNP U. (C) Anti-eIF3g (5 $\mu \mathrm{g})$ and anti-hnRNP U (5 $\mu \mathrm{g})$ antibody-immunoprecipitated proteins were analyzed by western blotting with anti-HSZFP36 antibody. Arrowheads indicate HSZFP36. (D) Anti-eIF3g (5 $\mu \mathrm{g})$ and anti- $\beta$-actin $(5 \mu \mathrm{g})$ antibody-immunoprecipitated proteins were analyzed by western blotting with anti- $\beta$-actin antibody. Arrowheads indicate $\beta$-actin. (E) Results of western blotting from the in vitro crosslinking. The nuclear extract from Bcap37/Tet-on-eIF3g cells was crosslinked by DSS, subject ed to 8\% SDS-PAGE and electrotransferred onto a polyvinylidene fluoride membrane, followed by incubation with antibodies specific for eIF3g, hnRNP U, HSZFP36 and $\beta$-actin. Arrowheads indicate larger complex bands; arrows indicate smaller complex bands. (F) GST-pulldown results. GST or GST-eIF3g proteins were incubated with nuclear lysates of Bcap37 cells and glutathione beads; bound proteins were resolved by 10\% SDS-PAGE and detected by western blotting. hnRNP U, HSZFP36 and $\beta$-actin were pulled down by GST-eIF3g, with GST protein and GSH beads alone used as negative controls. Arrowheads indicate eIF3g in the Bcap37 cells; arrows indicate the GST-eIF3g protein band. eIF3g, eukaryotic translation initiation factor 3 subunit g; hnRNP U, heterogeneous nuclear ribonucleoprotein U; GST, glutathione S-transferase; GSH, glutathione.

$>170 \mathrm{kDa}$ in molecular weight. The larger band was also detected by the anti-hnRNP U antibody, whereas the smaller band was detected by the anti-HSZFP36 or anti- $\beta$-actin antibodies. The results of the GST-pulldown assay showed that hnRNP U, HSZFP36 and $\beta$-actin were pulled down by the GST-eIF3g proteins (Fig. 3F). These results supported the interaction between eIF3g and hnRNP U, HSZFP36 or $\beta$-actin, and suggested that eIF3g, HSZFP36 and $\beta$-actin may form a protein complex.

Co-localization of eIF3g and its interacting proteins, HSZFP36 and $\beta$-actin, by immunofluorescent staining. The subcellular 
A
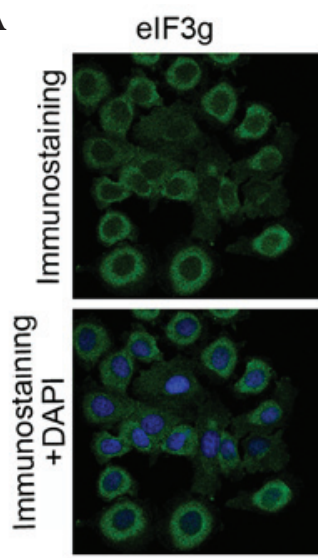

$\mathbf{B}$

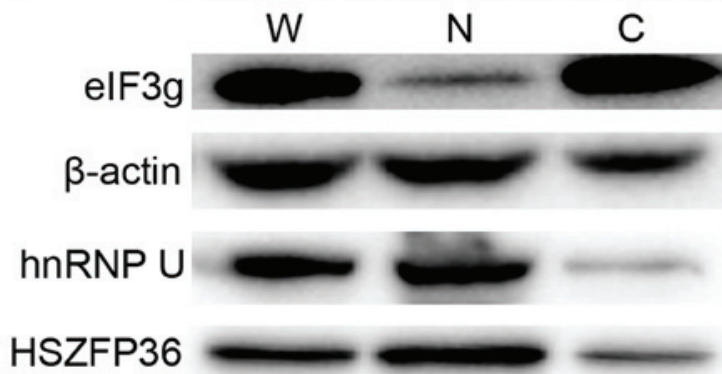

elF3g

HSZFP36

actin
hnRNP U
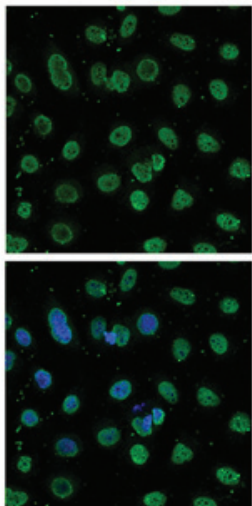

HSZFP36
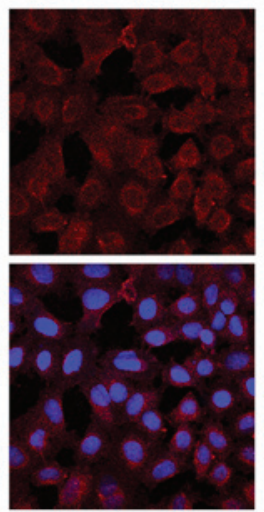

$\mathrm{C}$
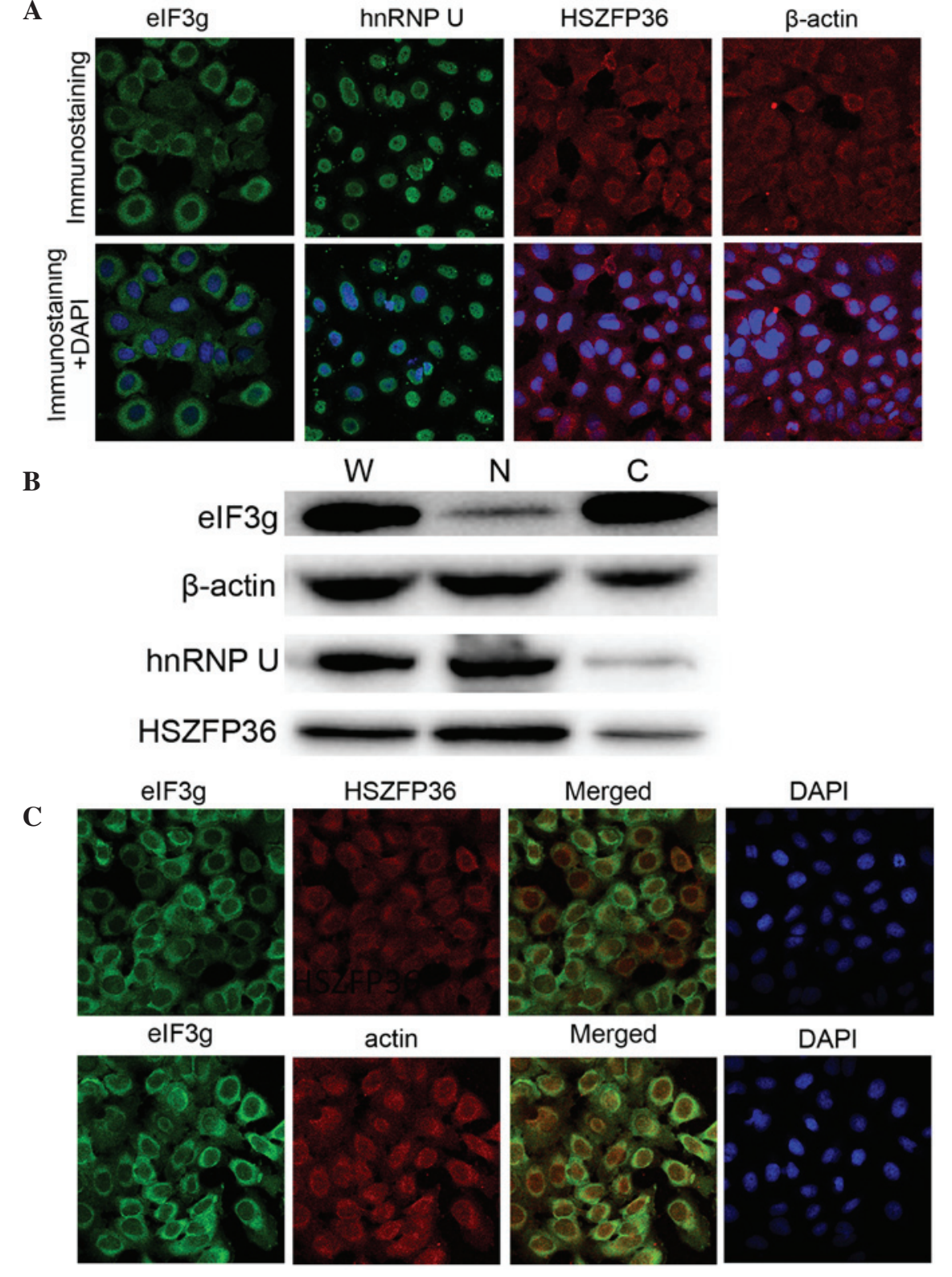

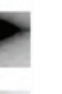

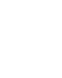

Figure 4. Co-localization of eIF3g, hnRNP U, HSZFP36 and $\beta$-actin. (A) Bcap37/Tet-on-eIF3g cells were incubated with anti-eIF3g, anti-hnRNP U, anti-HSZFP36 and anti- $\beta$-actin antibodies, respectively (magnification, x100). (B) Results of western blot analysis. Extracts from Bcap37/Tet-on-eIF3g cells were probed with anti-eIF3g, anti-hnRNP U, anti-HSZFP36 and anti- $\beta$-actin antibodies, respectively. (C) Co-localization of eIF3g, HSZFP36 and $\beta$-actin in the nuclei (magnification, x100). eIF3g, eukaryotic translation initiation factor 3 subunit g; hnRNP U, heterogeneous nuclear ribonucleoprotein U; W, whole cell lysate; C, cytoplasmic fraction; N, nuclear fraction.

distributions of eIF3g, hnRNP U, HSZFP36 and $\beta$-actin were examined by immunofluorescent staining and confocal microscopy. The cells incubated with anti-eIF3g antibody showed strong fluorescent signals in the cytoplasm and weak fluorescent signals in the nucleus. Similar patterns of fluorescence were observed in the cells incubated with anti-HSZFP36 and anti- $\beta$-actin antibodies. In parallel, fluorescence was predominantly observed in the nuclei of cells incubated with anti-hnRNP U antibody (Fig. 4A). The subcellular distributions of these four proteins were also confirmed by western blotting with the fractionated cytosolic and nuclear protein samples (Fig. 4B).

As shown in Fig. 4C, confocal microscopy revealed the co-localization of eIF3g with HSZFP36 and $\beta$-actin in the nucleus, although the intensities of the signals varied due to the different abundance of the proteins in the nucleus.

\section{Discussion}

The results of the present study demonstrated for the first time, to the best of our knowledge, the nuclear location of eIF3g, and its interactions with nuclear and cytoskeleton proteins. EIF3g is known to contain an RNA recognition motif (RRM) in its C-terminal region (320 amino acids). This mediates the interaction between the $43 \mathrm{~S}$ pre-initiation complex and the eIF4F-bound mRNA, leading to the formation of the translation initiation factor complex (2). It has been demonstrated that eIF3g is involved in the complex formation by interacting with eIF3b, eIF3i, eIF4B and RNA. There is also evidence showing that eIF3g (p44) interacts with eIF3a (p170) and binds to rRNA via the RRM domain (16). In addition, eIF3g has been suggested to be involved in the translational reinitiation process 
of cauliflower mosaic virus (CaMV) polycistronic mRNA and the yeast GCN4 mRNA $(17,18)$. In CaMV-infected plant cells, transactivator/viroplasmin (TAV) binds to the plant eIF3 via the eIF3g subunit to form TAV-eIF3-40S and TAV-eIF3-80S complexes, allowing the translation of polycistronic mRNAs by reinitiation (17). The centre of eIF3g, a fragment spanning amino acid residues 66-173, interacts with TAV or eIF4B, thus eIF4B can preclude the formation of the TAV/eIF3 complex via competition with TAV for eIF3g binding $(19,20)$.

In addition to its role in translation initiation/reinitiation, eIF3g may be involved in the cytoskeletal network and apoptosis. The cytoskeletal protein, erythroid protein 4.1 (4.1R) is reported to bind to eIF3g (p44) (21), and the evolutionary conserved protein, pelota, is reported to interact with eIF3g in the cytoskeleton (10). The involvement of eIF3g (p42) in apoptosis was observed in a proteomic study and confirmed using a knockdown assay, demonstrating that RNAi-induced eIF3g suppression inhibits the apoptotic process (22). The interaction between the N-terminus of eIF3g and apoptosis-inducing factor, a caspase-independent apoptotic factor, leads to the inhibition of protein synthesis during apoptosis (23). Previously, eIF3g was found to be cleaved by caspases during apoptosis, and the cleaved $\mathrm{N}$-terminus was translocated to the nucleus, showing a high level of DNase activity (11).

In our preliminary study, it was found that the increased expression of eIF3g in tumor cells correlates with lymph node metastasis and drug resistance in patients with breast cancer, and that the enforced expression of eIF3g in breast cancer cells significantly enhances proliferation, migration and invasion in vitro. In the present study, it was demonstrated that eIF3g can translocate into the nuclei of breast cancer cells, and that hnRNP U, HSZFP36 and $\beta$-actin can interact with eIF3g in the nucleus. As nuclear factors may exert a marked effect on the modulation of gene expression, which may lead to significant phenotypic changes including cell growth, even in small quantities, trace quantities of such nuclear eIF3g may be important to the behavior of the cells. These data provided clues to further investigate the molecular mechanisms underlying the tumor-promoting properties of overexpressed eIF3g in cancer cells.

Studies on the nuclear matrix-associated hnRNP U protein have shown that it is important in DNA repair, telomere length regulation, chromatin remodeling and gene expression regulation at the transcriptional and post-transcriptional levels (24-26). hnRNP $U$ is involved in the transcriptional activation of certain genes when cooperating with DNA topoisomerase II (27). It is also a potent regulator of nuclear ribonucleoprotein particles in diverse gene expression pathways (28), and enhances the expression of specific genes by stabilizing mRNA (29). Notably, compared with normal colonic epithelium, hnRNP U shows increased nuclear expression in colorectal cancer tissue (30). HSZFP36 is a member of the zinc finger protein family and is located predominantly in the nucleus, acting as a transcriptional regulator (31). Its roles in physiological/pathological conditions has not been investigated, however, studies on the functions of nuclear actin have shown that it is implicated in diverse nuclear activities, including chromatin remodeling, transcription regulation and nucleocytoplasmic trafficking (32). In HeLa cells, actin and hnRNP U cooperate with histone acetyltransferase PCAF in promoting transcription by RNA polymerase II $(33,34)$. Whether the interactions between eIF3g and these proteins have direct or indirect effects on the expression of genes responsible for the development and/or progression of breast cancer remains to be elucidated, however, nuclear eIF3g may act as an important molecule in assisting or inhibiting the functions of these interacting proteins. In the present study, further functional analysis of the proteins using PredictProtein revealed the possibility that eIF3g regulates gene expression at the transcriptional and post-transcriptional levels (Table II). Further investigations are required to identify the binding sites of eIF3g to hnRNP U, HSZFP36 and $\beta$-actin, to determine whether, and in what manner, eIF3g binds to DNA and to elucidate how nuclear eIF3g promotes tumor growth.

In conclusion, the data in the present study revealed the novel finding that eIF3g interacted with hnRNP U, HSZFP36 and $\beta$-actin in the nucleus. The present study identified these proteins as eIF3g-interacting proteins, however, whether the interaction between eIF3g and these proteins is direct or indirect remains to be elucidated. A detailed analysis of the eIF3g interaction with hnRNP U, HSZFP36 and $\beta$-actin may provide novel insight into the functions of eIF3g. The present study provided a basis for further investigation on the association between the altered expression of eIF3g in breast cancer cells and the malignant phenotypes of breast cancer cells, including invasion and metastasis, and may also provide more potential novel markers and/or therapeutic targets for breast cancer.

\section{Acknowledgements}

This study was supported by the National Natural Science Foundation of China (grant no. 81172516) and the Science and Technology Project (grant no. 2013B02) of Hangzhou Municipal Health Bureau, China. The authors would like to thank Dr Hao Zhu of the Department of Clinical Laboratory Sciences, University of Kansas Medical Center (Kansas City, KS, USA) for his assistance, comments and suggestions in manuscript preparation.

\section{References}

1. Silvera D, Formenti SC and Schneider RJ: Translational control in cancer. Nat Rev Cancer 10: 254-266, 2010.

2. Dong ZZ and Zhang JT: Initiation factor eIF3 and regulation of mRNA translation, cell growth, and cancer. Crit Rev Oncol Hematol 59: 169-180, 2006.

3. Hershey JW: Regulation of protein synthesis and the role of eIF3 in cancer. Braz J Med Biol Res 43: 920-930, 2010.

4. Buttitta F, Martella C, Barassi F, Felicioni L, Salvatore S, Rosini S, D'Antuono T, Chella A, Mucilli F, Sacco R, et al: Int6 expression can predict survival in early-stage non-small cell lung cancer patients. Clin Cancer Res 11: 3198-3204, 2005.

5. Cappuzzo F, Varella-Garcia M, Rossi E, Gajapathy S, Valente M, Drabkin H and Gemmill R: MYC and EIF3H coamplification significantly improve response and survival of non-small cell lung cancer patients (NSCLC) treated with gefitinib. J Thorac Oncol 4: 472-478, 2009.

6. Savinainen KJ, Helenius MA, Lehtonen HJ and Visakorpi T: Overexpression of EIF3S3 promotes cancer cell growth. Prostate 66: 1144-1150, 2006.

7. Lei YX, Wei L, Wang M, Wu GR and Li M: Malignant transformation and abnormal expression of eukaryotic initiation factor in bronchial epithelial cells induced by cadmium chloride. Biomed Environ Sci 21: 332-338, 2008. 
8. Zhang L, Pan X and Hershey JW: Individual overexpression of five subunits of human translation initiation factor eIF3 promotes malignant transformation of immortal fibroblast cells. J Biol Chem 282: 5790-5800, 2007.

9. Umar A, Kang H, Timmermans AM, Look MP, Meijer-van Gelder ME, den Bakker MA, Jaitly N, Martens JW, Luider TM, Foekens JA and Pasa-Tolić L: Identification of a putative protein profile associated with tamoxifen therapy resistance in breast cancer. Mol Cell Proteomics 8: 1278-1294, 2009.

10. Burnicka-Turek O, Kata A, Buyandelger B, Ebermann L, Kramann N, Burfeind P, Hoyer-Fender S, Engel W and Adham IM: Pelota interacts with HAX1, EIF3G and SRPX and the resulting protein complexes are associated with the actin cytoskeleton. BMC cell Biol 11: 28, 2010

11. Kim JT, Lee SJ, Kim BY, Lee CH, Yeom YI, Choe YK, Yoon DY, Chae SK, Kim JW, Yang Y, et al: Caspase-mediated cleavage and DNase activity of the translation initiation factor 3, subunit G (eIF3g). FEBS Lett 587: 3668-3674, 2013.

12. Zhu F, Wang Y, Zeng S, Fu X, Wang L and Cao J: Involvement of annexin A1 in multidrug resistance of K562/ADR cells identified by the proteomic study. Omics 13: 467-476, 2009.

13. Li C, Chen L, Ye J, Zhang X and Cao J: Establishment of breast cancer cell models with inducible d-ifferential eIF3g expressions. Xi Bao Sheng Wu Xue Za Zhi 34: 1226-1231, 2012 (In Chinese).

14. Suzuki K, Bose P, Leong-Quong RY, Fujita DJ and Riabowol K REAP: A two minute cell fractionation method. BMC Res Notes 3: 294, 2010.

15. Cokol M, Nair R and Rost B: Finding nuclear localization signals. EMBO Rep 1: 411-415, 2000

16. Block KL, Vornlocher HP and Hershey JW: Characterization of cDNAs encoding the p44 and p35 subunits of human translation initiation factor eIF3. J Biol Chem 273: 31901-31908, 1998.

17. Park HS, Himmelbach A, Browning KS, Hohn $\mathrm{T}$ and Ryabova LA: A plant viral 'reinitiation' factor interacts with the host translational machinery. Cell 106: 723-733, 2001.

18. Cuchalova L, Kouba T, Herrmannová A, Dányi I, Chiu WL and Valásek L: The RNA recognition motif of eukaryotic translation initiation factor $3 \mathrm{~g}$ (eIF3g) is required for resumption of scanning of posttermination ribosomes for reinitiation on GCN4 and together with eIF3i stimulates linear scanning. Mol Cell Biol 30 4671-4686, 2010.

19. Park HS, Browning KS, Hohn T and Ryabova LA: Eucaryotic initiation factor 4B controls eIF3-mediated ribosomal entry of viral reinitiation factor. EMBO J 23: 1381-1391, 2004

20. Ryabova L, Park HS and Hohn T: Control of translation reinitiation on the cauliflower mosaic virus (CaMV) polycistronic RNA. Biochem Soc Trans 32: 592-596, 2004.

21. Hou CL, Tang Cj, Roffler SR and Tang TK: Protein 4.1R binding to eIF3-p44 suggests an interaction between the cytoskeleta network and the translation apparatus. Blood 96: 747-753, 2000.
22. Machuy N, Thiede B, Rajalingam K, Dimmler C, Thieck O, Meyer TF and Rudel T: A global approach combining proteome analysis and phenotypic screening with RNA interference yields novel apoptosis regulators. Mol Cell Proteomics 4: 44-55, 2005

23. Kim JT, Kim KD, Song EY,Lee HG, Kim JW, Kim JW, Chae SK, Kim E, Lee MS, Yang Y and Lim JS: Apoptosis-inducing factor (AIF) inhibits protein synthesis by interacting with the eukaryotic translation initiation factor 3 subunit p44 (eIF3g). FEBS Lett 580: 6375-6383, 2006.

24. Carpenter B, MacKay C, Alnabulsi A, MacKay M, Telfer C, Melvin WT and Murray GI: The roles of heterogeneous nuclear ribonucleoproteins in tumour development and progression. Biochim Biophys Acta 1765: 85-100, 2006.

25. Ford LP, Wright WE and Shay JW: A model for heterogeneous nuclear ribonucleoproteins in telomere and telomerase regulation. Oncogene 21: 580-583, 2002.

26. Göhring F and Fackelmayer FO: The scaffold/matrix attachment region binding protein hnRNP-U (SAF-A) is directly bound to chromosomal DNA in vivo: A chemical cross-linking study. Biochemistry 36: 8276-8283, 1997.

27. Kawano S, Miyaji M, Ichiyasu S, Tsutsui KM and Tsutsui K: Regulation of DNA Topoisomerase IIbeta through RNA-dependent association with heterogeneous nuclear ribonucleoprotein U (hnRNP U). J Biol Chem 285: 26451-26460, 2010.

28. Xiao R, Tang P, Yang B, Huang J, Zhou Y, Shao C, Li H, Sun H, Zhang Y and Fu XD: Nuclear matrix factor hnRNP U/SAF-A exerts a global control of alternative splicing by regulating U2 snRNP maturation. Mol Cell 45: 656-668, 2012.

29. Yugami M, Kabe Y, Yamaguchi Y, Wada T and Handa H: hnRNP-U enhances the expression of specific genes by stabilizing mRNA. FEBS Lett 581: 1-7, 2007.

30. Hope NR and Murray GI: The expression profile of RNA-binding proteins in primary and metastatic colorectal cancer: Relationship of heterogeneous nuclear ribonucleoproteins with prognosis. Hum Pathol 42: 393-402, 2011.

31. Huebner K, Druck T, Croce CM and Thiesen HJ: Twenty-seven nonoverlapping zinc finger cDNAs from human T cells map to nine different chromosomes with apparent clustering. Am J Hum Genet 48: 726-740, 1991.

32. Bettinger BT, Gilbert DM and Amberg DC: Actin up in the nucleus. Nat Rev Mol Cell Biol 5: 410-415, 2004.

33. Kukalev A, Nord Y, Palmberg C, Bergman T and Percipalle P: Actin and hnRNP U cooperate for productive transcription by RNA polymerase II. Nat Struct Mol Biol 12: 238-244, 2005.

34. Obrdlik A, Kukalev A, Louvet E, Farrants AK, Caputo L and Percipalle P: The histone acetyltransferase PCAF associates with actin and hnRNP U for RNA polymerase II transcription. Mol Cell Biol 28: 6342-6357, 2008. 